HISTORIA

\title{
Raíces históricas, sociales y epidemiológicas de la tuberculosis en Bogotá, Colombia
}

\author{
Alvaro Javier Idrovo \\ Instituto Nacional de Salud Pública, Cuernavaca, México.
}

\begin{abstract}
El entendimiento completo de la dinámica epidemiológica actual de la tuberculosis en una sociedad requiere una aproximación holística. En este artículo se resume el comportamiento de la tuberculosis en Bogotá, Colombia, a través del tiempo. En periodos prehispánicos, la frecuencia de la enfermedad fue baja. Durante la Conquista y la Colonia se observó un incremento moderado de la tuberculosis pulmonar y abdominal. Hacia 1870 se inició un acentuado incremento de los casos de tuberculosis pulmonar, asociado con el proceso acelerado de la urbanización de la ciudad. Desde la segunda década del siglo XX, la tuberculosis pulmonar se convirtió en una enfermedad endémica. Después de 1920, la frecuencia fue relativamente estable hasta la década del 70, cuando se presentó un descenso; sin embargo, en los últimos años volvió a aparecer un aumento en el número de casos. La presencia de la tuberculosis en cada periodo se asocia con eventos sociales y ambientales.
\end{abstract}

Palabras clave: tuberculosis, agricultura, urbanización, industria, Colombia.

Historical, social and epidemiological roots of tuberculosis in Bogotá, Colombia

Understanding the current epidemiologic dynamic of tuberculosis (TB) in any society requires a holistic approach. In the current paper, the history and behavior of the TB is summarized for Bogotá, Colombia. In prehispanic periods the occurrence of TB was low. During the Conquest and the Colony periods, a moderate increase of pulmonary and abdominal TB was observed. In the 1870s, a great increase in cases of pulmonary TB was associated with the accelerated urbanization process. Since the 1920s, the occurrence of pulmonary TB shifted to the status of an endemic disease. After 1920, its occurrence was relatively steady until the 1970s, when its occurence greatly decreased. More recently, however, an increase in case numbers has been observed. The occurrence of TB in each period is associated with clearly defined social and environmental phenomena.

Key words: tuberculosis, agriculture, urbanization, industry, Colombia.

La tuberculosis es una enfermedad que ha acompañado al hombre, probablemente, desde su aparición, y que ha servido como trazadora de las cada vez más complejas relaciones entre el ambiente, las sociedades y la salud humana. Puede ser causada por Mycobacterium tuberculosis, Mycobacterium bovis, Mycobacterium africanum, Mycobacterium microti y Mycobacterium canettii

$\overline{\text { Correspondencia: }}$

Alvaro J. Idrovo, Centro de Investigación en Salud Poblacional, Instituto Nacional de Salud Pública, Avenida Universidad 655, CP 62508, Colonia Santa María Ahuacatitlán, Cuernavaca, Morelos, México.

idrovoaj@hotmail.com; ajidrovo@espm.insp.mx

Recibido:12/04/04; aceptado: 07/10/04
(1), denominados en conjunto como "complejo $M$. tuberculosis". Diversos trabajos en todo el mundo han mostrado que el estudio histórico de la tuberculosis puede proveer un mejor marco analítico para entender la dinámica epidemiológica de la enfermedad que únicamente el enfoque individualista propio de las disciplinas biomédicas y conductuales (2). En el presente artículo se revisa cómo ha sido el comportamiento de la tuberculosis en Bogotá a través de la historia y se señalan algunas posibles líneas futuras de investigación sobre el tema. Si bien el trabajo enfoca la enfermedad en la capital colombiana, sus hallazgos pueden servir como punto de partida para mejorar el entendimiento de la epidemiología 
social e histórica de la tuberculosis en todo el territorio nacional.

La historia de Bogotá se divide en cuatro grandes periodos, que corresponden con los cambios más significativos de la frecuencia de tuberculosis. Se inicia con las evidencias arqueológicas y antropológicas que permiten hacer una aproximación a la presentación de la tuberculosis en las poblaciones prehispánicas; luego, se analiza cómo ocurrió la mezcla de los patrones epidemiológicos de la tuberculosis de origen americano y europeo durante la Conquista y la Colonia; posteriormente, se señalan los procesos sociales involucrados en el inicio de la epidemia de tuberculosis en Bogotá hacia finales del siglo $\mathrm{XIX}$ y las primeras dos décadas del siglo XX, y se finaliza con una descripción del comportamiento de la enfermedad desde aquellos tiempos hasta la actualidad. En el cuadro 1 se presenta un resumen de los perfiles epidemiológicos de la tuberculosis en los periodos estudiados.

El presente análisis pretende no caer en un enfoque nihilista ni pragmático radical (3), sino más bien presentar un equilibrio entre éstos, de manera que se logre entender mejor la multicausalidad de la enfermedad. Es decir, no se tiene a priori una postura que apoye la necesidad de cambios sociales profundos (enfoque nihilista), ni una postura que señale las acciones médicas individuales relacionadas con el diagnóstico y el tratamiento (enfoque pragmático radical) como las responsables de la disminución de la frecuencia de la tuberculosis. La aproximación usada en este trabajo parte de la tesis de McKeown (4) e incorpora elementos de las críticas posteriores resultantes de los estudios empíricos que la refutaban (5), buscando identificar las particularidades del fenómeno en la capital colombiana.

\section{Tuberculosis prehispánica}

Existen evidencias irrefutables de la existencia de la tuberculosis en el Nuevo Mundo desde antes del descubrimiento de América. Se han encontrado lesiones compatibles con tuberculosis en Canadá (6), Estados Unidos (7-11), Colombia (12-15), Perú y Chile (16). Sin embargo, la prueba reina fue la identificación de segmentos de ADN de $M$. tuberculosis en lesiones de restos humanos encontrados en el norte de Chile y Perú $(17,18)$. En Colombia, los hallazgos de lesiones compatibles con tuberculosis en un individuo proveniente de La Mesa de los Santos, Santander, con imágenes radiológicas de dos lesiones calcificadas en la región superoanterior de la cavidad torácica izquierda (12), en la que recientemente se identificó ADN de $M$. tuberculosis (13), los encontrados en siete sujetos de un cementerio prehispánico muisca en Soacha (14) y los observados en los restos óseos de individuos del departamento del Cauca sugestivos de tuberculosis (15) constituyen las evidencias hasta ahora disponibles. Estos pocos casos no indican necesariamente una baja frecuencia entre las poblaciones aborígenes colombianas; más bien señalan las dificultades de estudiar "muestras sesgadas" en los estudios paleopatológicos $(19,20)$.

De acuerdo con un análisis previo (21), los posibles determinantes de la presencia de la tuberculosis en la población prehispánica de Bogotá se encuentran estrechamente relacionados con la aparición de la agricultura. La relación entre la agricultura y las enfermedades infecciosas es el patrón más frecuentemente observado en las poblaciones prehistóricas (22). Sin embargo, es posible que esta asociación no se haya observado en otras regiones del país con importante desarrollo agrícola, como la zona del Sinú donde

Cuadro 1. Probable comportamiento de la tuberculosis en Bogotá a través de su historia.

\begin{tabular}{lll}
\hline Periodo & Mycobacterium tuberculosis & Mycobacterium bovis \\
\hline Prehispánico & Frecuencia baja & Ausente \\
Conquista y Colonia & Incremento & Incremento \\
$1870-1920$ & Incremento acentuado & Decremento \\
1921 en adelante & Decremento/reemergencia & Notorio decremento \\
\hline
\end{tabular}


se combinaba el cultivo de tubérculos, principalmente yuca, con la pesca como sus actividades de subsistencia $(23,24)$. Existen evidencias en el territorio panameño, donde habitaron pueblos íntimamente relacionados con la cultura del Sinú, que indican que el desarrollo agrícola no favoreció el incremento de la frecuencia de enfermedades infecciosas (25).

El territorio de la actual Bogotá fue habitado por los grupos agroalfareros de la llamada Cultura Herrera, hasta que fueron desplazados 0 absorbidos por los muiscas hacia los siglos IX o $X$ d.C. Éstos provenían de la Costa Atlántica venezolana o de las tierras bajas del oriente suramericano; eran pequeños agricultores asociados en poblaciones nucleadas que no llegaban a conformar grandes urbes. Sus cultivos principales eran de maíz, papa, cubios, fríjol, batata, yuca, arracacha, ají, maní, piña y algunos frutales (26). Es bien conocido que no sólo es necesario que exista un suficiente número de individuos para que la enfermedad se mantenga prevalente en una población; se requieren otras condiciones como las malas condiciones higiénicas, la convivencia con animales portadores de Mycobacterium, algunos factores debilitantes y la inmunosupresión para que se desarrolle la tuberculosis.

Los muiscas eran un pueblo con muy buenas costumbres de aseo personal, entre quienes se observaban algunas veces alcantarillados rudimentarios (27), relacionados con el culto que le tenían al agua. La fauna asociada con sus asentamientos estaba constituida por curíes (Cavia porcellus), venados, guaguas, comadrejas, cusumbos, palomas, ratones, conejos, zorros y pecaríes $(26,28)$; el primero era el más importante por ser el principal animal doméstico. Los curíes pueden infectarse con Mycobacterium por inhalación o ingestión de esputos e, incluso, desarrollar la enfermedad. Los procesos involucrados en esta transmisión, estudiados hacia finales del siglo XIX y comienzos del siglo $X X$, fueron descritos por diversos investigadores, entre los que sobresalen Flügge, Calmette, Guérin, Koch, Strauss, Rothe, Raymond, Römer y Feyerabend $(29,30)$. Infortunadamente, es muy difícil corroborar el papel que jugaron los curíes en la transmisión de la tuberculosis en las poblaciones prehispánicas, ya que de haber sucedido es posible que las lesiones en los humanos hubieran sido en órganos blandos que rara vez se conservan hasta nuestros días.

Con respecto a los factores debilitantes y la inmunosupresión, la desnutrición es la condición que pudo haber estado relacionada. La alimentación muisca estaba fundamentada en el maíz, los fríjoles, las raíces, las papas, las frutas y la carne de venados, tórtolas, conejos, curíes, palomas y perdices (31). Clásicamente, un elevado número de dientes cariados y los trastornos del hueso cortical se asocian con una alta ingestión de carbohidratos; estos signos se observan frecuentemente en los restos de las mujeres muiscas (14). Este hecho permite suponer que la dieta con proteína animal era privilegio de los hombres, principalmente, de posiciones sociales superiores. Sin embargo, los estudios con los isótopos $\delta 13 \mathrm{C}$ de colágeno y apatita, y $\delta 15 \mathrm{~N}$ del colágeno que permiten determinar el consumo diferencial de vegetales y animales, identificaron que también existía un consumo alto de proteína animal entre las madres y los niños en edades de amamantamiento (31).

Por estas razones, si bien la infección por Mycobacterium pudo tener una alta prevalencia entre los muiscas, la enfermedad posiblemente se presentó con mayor frecuencia entre las mujeres. Esto pudo deberse a las condiciones inapropiadas para presentar una morbimortalidad elevada asociada con la enfermedad. Futuros estudios podrían incluir la amplificación del fragmento IS6110 del ADN y la subunidad B de la ARN polimerasa para identificar la infección por microorganismos del complejo $M$. tuberculosis (32), tal como se hizo con el individuo guane, proveniente de Santander, recientemente estudiado por Sotomayor et al. (13).

\section{La tuberculosis oculta (1538-1870)}

No hay dudas de la presencia de la tuberculosis en territorio bogotano durante los periodos de la Conquista y la Colonia, aunque existen grandes dificultades para conocer su comportamiento debido a la ausencia de evidencia empírica y a las escasas y limitadas descripciones de los 
cronistas y médicos de la época. El fenómeno social más sobresaliente de la Conquista fue el encuentro, a veces amistoso y muchas otras veces bélico, de individuos europeos con aborígenes americanos. El resultado de este encuentro, en términos de la presencia de la tuberculosis, fue el choque de dos patrones epidemiológicos que con el paso del tiempo terminó en el predominio del perfil europeo durante la Colonia y la primera República. El año que marca el encuentro de los dos perfiles epidemiológicos en Bogotá fue 1538, el año de su fundación. Para entender mejor este encuentro es necesario revisar la presencia de la tuberculosis en los lugares de origen de los conquistadores y los colonizadores.

Durante gran parte de los periodos de la Conquista, la Colonia y la primera República, la tuberculosis en España y otros países de Europa era considerada como una enfermedad endémica que afectaba a buena parte de la población (33). Diversos análisis históricos señalan que las infecciones fueron causadas por $M$. tuberculosis y $M$. bovis; la primera era predominante en las ciudades y la segunda en las zonas rurales. Las evidencias también indican que la primera se asociaba con lesiones pulmonares, mientras la segunda con lesiones extrapulmonares, y que los más afectados por M. bovis eran los niños (3).

La tuberculosis bovina ha estado presente en Europa con frecuencias nada despreciables hasta hace pocas décadas $(34,35)$. La enfermedad, reconocida o no por los médicos veterinarios de la época, debió ser tan importante que fue tratada en varios libros especializados de comienzos del siglo XIX (36). Dado que la historia general de la enfermedad indica que la tuberculosis bovina precedió a la tuberculosis causada por $M$. tuberculosis (37), es posible pensar que muchos de los conquistadores tuvieron la infección e, incluso, la enfermedad establecida al llegar a tierras americanas. Sin embargo, es mucho más probable que $M$. bovis hubiese llegado a América con el ganado bovino que fue introducido al Nuevo Mundo en los primeros años de la Conquista.

Para tener evidencia empírica que apoye la presencia de tuberculosis bovina durante este periodo y los siguientes, se podría realizar la espoligotipificación de diferentes razas bovinas actuales (38), que incluya descendientes del orejinegro andaluz traído a América desde los inicios de la Conquista (39). Esta técnica ha permitido identificar las relaciones del ganado de Inglaterra y Australia, Canadá, Irán y Argentina, e identificar diferencias con el procedente de Francia (38). Para confirmar esta hipótesis, es de esperar que los resultados hallados en Colombia muestren relación principalmente con el perfil español, caracterizado por una alta frecuencia $(46 \%)$ del espoligotipo Sp7 (40).

De otro lado, en España la tuberculosis causada por M. tuberculosis debió seguir los patrones encontrados en países vecinos como Francia, donde la agricultura, la urbanización descontrolada, la industrialización y otros factores relacionados fueron factores que favorecieron su propagación (35). Sin embargo, algunas características de la España de aquella época pudieron darle especificidad al comportamiento de la enfermedad. El crecimiento demográfico de la población española fue más lento que el ocurrido en otros países europeos (41); las ciudades, hasta más allá de la segunda mitad del siglo XIX, no tenían una adecuada planificación, especialmente las amuralladas, lo cual permitía que fueran prevalentes el hacinamiento y las malas condiciones sanitarias (42).

Por todo esto, si bien la tuberculosis empezó a disminuir de manera generalizada en Europa occidental desde la segunda mitad del siglo XIX (3), en España este proceso pudo ser más lento. La verificación de esta hipótesis será posible realizarla si se analizan los restos humanos de individuos de aquel periodo que se encuentran en los cementerios conservados hasta la actualidad mediante pruebas genéticas como la amplificación del fragmento IS6110 del ADN y la subunidad B de la ARN polimerasa (32). Son varias las preguntas que surgen en relación con este periodo para los investigadores de la historia social de la tuberculosis en Colombia, entre las que se pueden señalar: 1) ¿cuál es el efecto que tuvieron las guerra de independencia y, posteriormente, los conflictos bélicos internos durante el resto del siglo XIX sobre la frecuencia de la tuberculosis? y 2) 
¿será que tuvieron impactos similares a los observados en otros conflictos armados, como el franco-pruso de finales del siglo XIX (35)?

\section{La epidemia de tuberculosis pulmonar (1870-1920)}

Los hechos relacionados con el inicio de la epidemia de tuberculosis pulmonar en Bogotá ya se han estudiado previamente (43). Brevemente, a comienzos del siglo XIX Bogotá era una ciudad de una relativa gran extensión, aunque con una población de apenas 21.464 habitantes. Eran frecuentes las casas amplias, los templos y los conventos, por lo que el hacinamiento no era habitual en aquel tiempo. La población fue aumentando durante todo ese siglo, primero lentamente debido a la alta mortalidad ocasionada por las epidemias de viruela y las guerras civiles, y hacia las décadas finales del siglo de manera rápida. Para 1905, la población bogotana era cercana a los 100.000 habitantes (44), y ya se encuentra evidencia que señala el hacinamiento en que vivían las clases más pobres; mientras en el segundo piso de las casas vivían cómodamente las clases más adineradas, los primeros pisos eran utilizados como locales de chicherías o tiendas que, también, funcionaban como cocina, comedor y habitación de humanos y animales (45). Además, las condiciones de trabajo en la época podían tener efectos adversos sobre la salud; si bien la mayoría de los habitantes se dedicaba a labores no industriales, era frecuente el trabajo físico arduo y las largas jornadas laborales, incluso para la población infantil y de mujeres embarazadas (30).

Según los cronistas de la época, las condiciones sanitarias de la ciudad eran lamentables, debido a la inexistencia de adecuados servicios públicos de acueducto, alcantarillado y aseo $(46,47)$. La nutrición de los bogotanos era deficiente; se basaba en alimentos como chicha, caldos de agua-sal, mazamorra de harina de maíz con habas o coles y vísceras de animales, chorizos y longanizas (45). Estas últimas muchas veces eran consumidas en estado de descomposición, lo cual pudo favorecer la transmisión de M. bovis. El ganado bovino traído desde los primeros años de la Conquista pudo traer esta micobacteria desde Europa, y permitir su permanencia. No hay que olvidar que la carne para consumo humano no tuvo control sanitario en Bogotá hasta 1886 (48).

Al respecto existen algunas dudas generadas por el hallazgo de lesiones de Oesophagostomum radiatum en las vísceras animales (49), que podrían haber sido confundidas con las de tuberculosis bovina. Sin embargo, esta observación sólo se logró hacia 1915 y no es posible extrapolarla a los periodos previos. Un punto a favor de la existencia de la tuberculosis bovina en Bogotá es el hallazgo patológico de tubérculos abdominales en los habitantes de la ciudad; esto llamó en gran medida la atención de los médicos de la época, ya que observaban un patrón contrario al europeo: "En Europa la peritonitis sigue con frecuencia a una pleuresía; en Bogotá se observa lo contrario" (45). Debido a esto es posible pensar que la tuberculosis bovina sí existió en Bogotá y que, incluso, confirió protección cruzada contra la tuberculosis pulmonar (50).

Con base en los registros del Hospital San Juan de Dios que para la época atendía a cerca de $50 \%$ de los casos de tuberculosis de la ciudad (49), se puede conocer que entre 1875 y 1914 se inició la epidemia de tuberculosis pulmonar en Bogotá (43). La mortalidad por tuberculosis pulmonar se convirtió en la primera causa de muerte al pasar de ser cercana a $5 \%$ y llegar a tener proporciones de hasta $30 \%$ en sólo unos pocos años (49). La anatomía patológica de la época permitió identificar que cada vez con mayor frecuencia se seguía la teoría unicista de Bayle, Laennec y Louis, en la cual la infección inicial ocurría en los campos pulmonares inferiores; luego, los bacilos se ubicaban en los ápices y de allí se diseminaban hacia el resto del cuerpo. Esta alarmante situación propició que se crearan, en cumplimiento de la Ley 66 de 1916, pabellones especiales para tuberculosos en los hospitales más importantes del país (51).

\section{La endemia de tuberculosis pulmonar del siglo XX}

Después del rápido incremento de la frecuencia de tuberculosis antes descrito, la enfermedad se asentó de manera definitiva en la ciudad. Es así que para la segunda década del siglo $X X$ ya se consideraba una enfermedad endémica; en 1920, 
Pablo García Medina afirmaba que la tuberculosis se había extendido por todo el territorio nacional, especialmente de las costas hacia las regiones de clima templado y frío (52), entre las que se encontraba Bogotá. La situación llegó a ser tan alarmante que, desde 1924, los hospitales que recibían auxilios estatales estuvieron obligados a atender a los tuberculosos (53); debido a esta disposición, durante este tiempo el Hospital San Juan de Dios fue la principal institución prestadora de servicios a los tuberculosos capitalinos.

Sin embargo, los servicios allí ofrecidos eran limitados por lo que, entre 1930 y 1950, se crearon diversas instituciones encargadas de la atención y beneficencia a los enfermos que, obviamente, tuvieron acciones en los tuberculosos de Bogotá. En 1932 se creó la sección de lucha contra la tuberculosis que, en 1936, se convirtió en Departamento Nacional; en 1937 se organizó la Campaña Antituberculosa Nacional y se originaron los comités voluntarios contra la tuberculosis; en 1938 se fundó el Comité Femenino Antituberculoso de la Cruz Roja Nacional que el año siguiente cambió su nombre a Liga Antituberculosa Colombiana y, luego, se crearon los primeros hospitales antituberculosos en Bogotá, el de Santa Clara y el de San Carlos (51).

El primer hospital fue creado en 1942 con dineros públicos y fue donde se practicó un gran número de cirugías para el tratamiento de la tuberculosis durante las décadas siguientes (54). El Hospital de San Carlos, creado en 1948, era de carácter privado y tenía características excepcionales para su época; fue dotado con la mejor tecnología médica y construido con un arquitectura que seguía estrictamente los conocimientos científicos $(53,55)$. El tratamiento allí practicado consistía en hospitalización prolongada, reposo absoluto, alimentación regulada y el consumo de ácido $p$ aminosalicílico, hidracida del ácido isonicotínico y estreptomicina. Este tratamiento tuvo dos importantes modificaciones; en la década del 60 se introdujeron la rifampicina, el etambutol y la piracinamida, y en la década del 70 , después del descubrimiento de Madras, se estandarizó el tratamiento ambulatorio supervisado de corta duración (seis a nueve meses) lo cual propició que los sanatorios fueran desapareciendo (51).
Éste es el fundamento del tratamiento, denominado DOTS que persiste en la actualidad (56).

Hasta el momento no se ha hecho un análisis cuantitativo de los registros de morbilidad y mortalidad por tuberculosis en Bogotá durante este periodo; sin embargo, es consistente en los documentos de la época que la enfermedad tuvo una alta frecuencia (González G. Estado de la salud pública en Colombia. Primer Congreso Colombiano de Salud Pública. Medellín, 1962. p. 23-159 y Serpa F. Enfermedades transmisibles en Colombia. Primer Congreso Colombiano de Salud Pública. Medellín, 1962. p.184-91). Las condiciones que precipitaron la epidemia de tuberculosis en las décadas anteriores fueron las que facilitaron que la enfermedad se mantuviese con un carácter endémico en la ciudad durante cinco décadas, aproximadamente, y posteriormente empezara a disminuir. De esta manera, los fenómenos sociales relacionados fueron la urbanización no planificada y sin adecuados servicios públicos y, posteriormente, el desarrollo industrial. Los patrones observados en Bogotá no fueron, entonces, muy diferentes de los presentados en los países europeos y americanos (35,57-60). Es decir, que la frecuencia tendió a disminuir de manera acorde con el mejoramiento de las condiciones de vida de las clases trabajadoras (61).

En la segunda década del siglo XX se dieron importantes conquistas entre los trabajadores de las empresas más grandes de la ciudad. Gracias a la Ley 46 de 1918, y en respuesta a la epidemia de gripa que ese año azotó el país, se comenzaron a hacer mejoras a las viviendas de los proletarios; también se empezaron a observar los primeros servicios médicos empresariales (53), germen de la medicina del trabajo en Colombia. Hechos como éstos pueden considerarse los determinantes principales de la disminución de la frecuencia, junto a las acciones de vacunación y tratamiento médico, observado desde 1970 a 1997. Sin embargo, estas cifras son motivo de discusión, debido a que paralelamente también se disminuyó la detección de casos (62).

Actualmente, en Colombia el control de la tuberculosis se fundamenta en la detección de casos, el diagnóstico mediante técnicas de 
laboratorio y el tratamiento acortado supervisado gratuito para los enfermos. Este modelo, objeto de muchas críticas, ha permitido señalar que después de 1997 hay una tendencia al incremento de la frecuencia de sintomáticos respiratorios, baciloscopias positivas y mortalidad por tuberculosis (63). Durante estos años, la capital colombiana ha sido una de las regiones más afectadas por la tuberculosis en el país (64).

Las causas de estos cambios recientes en el comportamiento de la tuberculosis no han sido estudiadas ampliamente. Sin embargo, algunos estudios han empezado a explorar varios posibles determinantes:

1. La asociación tuberculosis-sida. Un argumento frecuente es el asociar la tuberculosis, principalmente extrapulmonar, con la infección con el VIH y el sida; en Colombia ya se tiene evidencia epidemiológica al respecto (65). Además, se ha observado que, en Bogotá, los pacientes con sida presentan una prevalencia de infección por $M$. tuberculosis que está entre $1,4 \%$ y $8 \%$ (66).

2. Resistencia a los medicamentos. Es bien conocido que, desde 1970, ha habido un incremento de micobacterias resistentes a algunos medicamentos, como la isoniacida, mientras se disminuye la resistencia a otros fármacos antituberculosos (67). Si bien estos cambios son ambivalentes, no pueden dejarse pasar desapercibidos dada la importancia que tienen para definir el tipo de tratamiento.

3. Efectos del nuevo sistema de salud. Ayala y Kroeger han sugerido que la implantación de la Ley 100 de 1993 pudo favorecer la disminución de la detección de tuberculosos, y que esto pudo ser debido, principalmente, a la falta de preparación en los municipios para los cambios (68).

4. Estigmatización de los tuberculosos. Los estudios realizados en Cali, quizá extrapolables en cierta medida a Bogotá, señalan que los individuos enfermos de tuberculosis son estigmatizados por la sociedad, y que el principal predictor de dicha conducta son las creencias sin fundamento científico sobre la transmisión de la tuberculosis (69).
5. Sistemas de conocimiento sobre la enfermedad. Otros estudios cualitativos han permitido identificar los pasos y las barreras que presentan los enfermos de tuberculosis en Cali, hasta acceder a los servicios de salud; entre los obstáculos, son de especial importancia las diferencias de los conocimientos de los profesionales de la salud y los pacientes (70).

6. Desplazados. La migración interna debida al conflicto armado en el país es un fenómeno social que, sin duda, tiene repercusiones sobre la salud de las poblaciones desplazadas, así como de las receptoras. Hasta ahora, no hay estudios específicos sobre los efectos del desplazamiento sobre la presencia de la tuberculosis en Bogotá, pero sí hay evidencias en otras ciudades que muestran que las condiciones de saneamiento y, en general, de vida de estas poblaciones son muy precarias (71); por tal razón, no es incoherente señalar que podría estar resurgiendo entre estos grupos una frecuencia elevada de tuberculosis.

\section{Conclusión}

Si bien es posible entender los cambios temporales en la frecuencia de tuberculosis mediante el número promedio de individuos "efectivamente contactados" por cada persona enferma (72), puede ser mucho más importante conocer el cómo y el porqué ocurrieron dichos contactos. Para ello, la única forma de comprenderlo es mediante abordajes amplios, como el presente, que permitan interpretar la compleja red causal involucrada en este proceso $(73,74)$; es decir, se debe tener en cuenta tanto los procesos biológicos como los sociales que ocurren en diferentes niveles de agregación y requieren aproximaciones diversas. El análisis de los estudios arqueológicos, históricos, sociales y epidemiológicos de los determinantes de la presencia de tuberculosis en Bogotá permite señalar que los determinantes han cambiado con el paso del tiempo. Mientras más se avanza en la historia, más complejas se tornan las relaciones sociales de las poblaciones humanas, lo cual hace más difícil entender las redes causales.

En los periodos prehispánicos, cuando la vida era relativamente sencilla, la explicación es muy 
simple: las actividades humanas asociadas con la agricultura permitieron que la tuberculosis se mantuviera dentro de la población aunque con una baja frecuencia. El encuentro de dos mundos durante la Conquista, la Colonia y los primeros años de la República ocasionó una mezcla de los patrones de comportamiento epidemiológico de la tuberculosis que, al final, llevó a un dominio del patrón europeo de infección por M. tuberculosis y M. bovis. Hacia finales del siglo XIX, la situación cambió radicalmente; probablemente, descendió la presencia de manifestaciones abdominales por $M$. bovis y se configuró una epidemia de tuberculosis pulmonar asociada con el proceso acelerado de urbanización de comienzos del siglo $X X$. Entre la tercera y la séptima década del siglo $\mathrm{XX}$, las precarias condiciones de las clases trabajadoras permitieron que se mantuviera una frecuencia relativamente constante; la mejoría paulatina de las condiciones de vida de los trabajadores durante el siglo XX y las acciones de aislamiento y tratamiento antimicrobiano de los enfermos fomentaron un descenso de la frecuencia de tuberculosis que se inició hacia 1970. En los últimos años, este perfil nuevamente parece cambiar y muestra un nuevo incremento de la frecuencia de la tuberculosis en la capital colombiana, de manera similar a lo observado en muchas regiones del mundo.

Como se puede apreciar, el tener sociedades cada vez más complejas en el territorio bogotano hizo que las redes causales hayan aumentando también su complejidad. Es por esto que resulta ilógico que haya quienes pretendan entender el comportamiento de la tuberculosis simplemente con aproximaciones biológicas (enfoque pragmático radical); para un entendimiento más real se requieren abordajes muchos más holísticos, sin llegar necesariamente al nihilismo, que involucren los diferentes puntos de vista de las disciplinas sociales. Un reto para los futuros investigadores de la historia social de la tuberculosis en Bogotá es la determinación del impacto de los posibles nuevos determinantes de la frecuencia actual y futura de la enfermedad, sin dejarse cegar por los importantes avances en la genómica que, sin duda, impregnarán y dominarán el conocimiento científico.

\section{Referencias}

1. Tiruviluamala $\mathbf{P}$, Reichman LB. Tuberculosis. Annu Rev Public Health 2002;23:403-26.

2. Gandy M, Zumla A. The resurgence of disease: social and historical perspectives on the "new" tuberculosis. Soc Sci Med 2002;55:385-96.

3. Fairchild AL, Oppenheimer GM. Public health nihilism vs pragmatism: history, politics, and the control of tuberculosis. Am J Public Health 1998;88:1105-17.

4. McKeown T. The modern rise of population. New York: Academic Press; 1976.

5. Szreter S. The importance of social intervention in Britain's mortality decline, 1850-1914: a re-interpretation of the role of public health. Soc Hist Med 1988;1:1-37.

6. Pfeiffer S. Paleopathology in an Iroquoian Ossuary, with special reference to tuberculosis. Am J Phys Anthropol 1984;65:181-9.

7. Means H. Roentgenological study of the skeletal remains of the prehistoric Mound Builder Indians of Ohio. Am J Roentgenol 1925;13:359-67.

8. Ritchie WA. Paleopathological evidence suggesting Precolumbian tuberculosis in New York State. Am J Phys Anthropol 1952;10:305-18.

9. Lichtor J, Lichtor A. Paleopathological evidence suggesting Precolumbian tuberculosis of the spine. $\mathrm{J}$ Bone Joint Surg 1957;39:1398-9.

10. Perzigian A, Widmer L. Evidence for tuberculosis in a prehistoric population. JAMA 1979;241:2643-6.

11. El-Najjar MY. Human treponematosis and tuberculosis: evidence from the New World. Am J Phys Anthropol 1979;51:599-618.

12. Correal G, Flórez I. Estudio de las momias guanes de La Mesa de los Santos (Santander, Colombia). Rev Acad Colomb Cienc 1992;18:183-90.

13. Sotomayor H, Burgos J, Arango M. Demostración de tuberculosis en una momia prehispánica colombiana por la ribotipificación del ADN de Mycobacterium tuberculosis. Biomédica 2004;24(Supl.):18-26.

14. Rodríguez JV. Acerca de la supuesta debilidad mental y física de los muiscas como posible causa de conquista y posterior extinción. Arqueología 1988; 1:42-6.

15. Rodríguez EL, Rodríguez CD, Díaz ML, González LC. Tuberculosis en poblaciones prehispánicas de "EI Morro de Tulcán" y "El Tambo-Alto del Rey" en el departamento del Cauca, Colombia. Infectio 2000;4:17.

16. Allison M, Mendoza D, Pezzia A. Documentation of a case of tuberculosis in Precolumbian America. Am Rev Respir Dis 1973;107:985-91.

17. Salo WL, Aufderheide AC, Holcomb TA. Identification of Mycobacterium tuberculosis DNA in a Pre-Columbian 
Peruvian mummy. Proc Natl Acad Sci USA 1994;91: 2091-6.

18. Arriaza BT, Salo W, Aufderheide AC, Holcomb TA. Pre-Columbian tuberculosis in northern Chile: molecular and skeletal evidence. Am J Phys Anthropol 1995;98: 37-45.

19. Waldron T. Counting the dead. The epidemiology of skeletal populations. Chichester: Wiley; 1994.

20. Roberts CA, Grauer A. Commentary: bones, bodies and representivity in the archaeological record. Int $\mathrm{J}$ Epidemiol 2001;30:109-10.

21. Idrovo AJ. Tuberculosis prehispánica en muiscas de la sabana de Bogotá. Rev Fac Med UN Col 1997;45: 55-9.

22. Armelagos GJ. Health and disease in prehistoric populations in transition. En: Swedlund AC, Armelagos GJ, editors. Disease in populations in transition. Anthropological and epidemiological perspectives. New York: Bergin and Garvey; 1990. p.127-44.

23. Bray W. Ancient food for thought. Nature 2000; 408:145-6.

24. Plazas C, Falchetti AM, Sáenz-Samper J, Archila J. La sociedad hidráulica Zenú. Estudio arqueológico de 2.000 años de historia en las llanuras del Caribe colombiano. Santafé de Bogotá: Colección bibliográfica Banco de la República; 1993.

25. Black FL. Infectious disease and evolution of human populations: the example of South American forest tribes. En: Swedlund AC, Armelagos GJ, editors. Disease in populations in transition. New York: Bergin \& Garvey; 1990. p.55-74.

26. Langebaek C. Mercados, poblamiento e integración entre los muiscas. Siglo XVI. Bogotá: Banco de la República; 1987. p.1-168.

27. Fernández de Piedrahita L. Historia general del Nuevo Reino de Granada. Tomo I. Bogotá: Editorial ABC; 1942. p.72.

28. Enciso BE. El ocaso del sol de los venados. Arqueología de rescate en la sabana de Bogotá. Rev Colomb Antropol 1993;30:149-82.

29. Calmette A. L'infection tuberculeuse spontanée du cobaye et du lapin. Ann Inst Pasteur 1933;50:148-60.

30. Solano N. Tuberculosis en Colombia: estudio sobre su etiología y profilaxis (tesis). Bogotá: Universidad Nacional; 1909.

31. Cárdenas F. Paleodieta y paleodemografía en poblaciones arqueológicas muiscas (sitios Las Delicias y Candelaria). Rev Colomb Antropol 1993;30:129-47.

32. Taylor GM, Goyal M, Legge AJ, Shaw RJ, Young D. Genotypic analysis of Mycobacterium tuberculosis from medieval human remains. Microbiology 1999;145:899904.
33. Nadal J. La población española. Barcelona: Ariel; 1975. p.149-61.

34. Benet JJ. Pour en finir avec la tuberculose. Bull Groupements Techniques Vet 2001;7:417-24.

35. Barnes DS. The making of a social disease: tuberculosis in nineteenth-century France. Berkeley: University of California Press; 1995.

36. Gutiérrez García JM. La tuberculosis bovina como zoonosis en la España contemporánea (1850-1950) (Tesis). Bellaterra: Universidad Autónoma de Barcelona; 2003.

37. Bates JH, Stead WW. The history of tuberculosis as a global epidemic. Med Clin North Am 1993;77:120517.

38. Hadad N, Ostyn A, Karoui C, Masselot M, Thorel MF, Hughes SL et al. Spoligotype diversity of Mycobacterium bovis strains isolated in France from 1979 to 2000. J Clin Microbiol 2001;39:3623-32.

39. Gómez A. De las dehesas de Andalucía a hoy. En: La ganadería en América Latina. Colombia. Bogotá:Tercer Mundo; 1976. p.125-7.

40. Aranaz A, Liebana E, Mateos A, Dominguez L, Vidal D, Domingo $O$ et al. Spacer oligonucleotide typing of Mycobacterium bovis strains from cattle and other animals: a tool for studying epidemiology of tuberculosis. J Clin Microbiol 1996;34:2734-40.

41. Tortella-Casares G. La economía española, 18001930. En: Tuñón de Lara M, editor. Historia de España. Barcelona: Editorial Labor; 1981. p.18.

42. Suriol J. Los ingenieros de caminos en la transformación urbana de las ciudades españolas a finales del siglo XIX. El caso de Barcelona. Scripta Nova. Rev Elect Geog Cienc Soc 2002; 6(120). Revista electrónica. Disponible en: http://www.ub.es/geocrit/sn/ sn-120.htm.

43. Idrovo AJ. Notas sobre el inicio de la epidemia de tuberculosis pulmonar en Bogotá (1870-1920). Biomédica 2001;21:216-23.

44. Fundación Misión Colombia. Vida cotidiana y cultura Aspectos demográficos. En: Historia de Bogotá. Tomo II - Siglo XIX. Bogotá: Salvat-Villegas Editores; 1989. p.9-56.

45. Fajardo C. Tuberculosis en el Hospital Civil de Bogotá (tesis). Bogotá: Universidad Nacional y Samper Matíz, 1896.

46. Camacho S. Mis memorias. Bogotá: s.e.; 1894. p.98-9.

47. Holton IF. La Nueva Granada: veinte meses en los Andes. Bogotá: Banco de la República: 1981. p.187.

48. Anónimo. Tesis presentada por Antonio María Olaechea en el examen general de veterinaria para optar el título de médico en este ramo. Anales de Instrucción Pública 1890;17:262-71. 
49. Isaza E. La tuberculosis en Bogotá - Profilaxis (tesis). Bogotá: Universidad Nacional y Arboleda \& Valencia; 1915.

50. Lietman T, Porco T, Blower S. Leprosy and tuberculosis: the epidemiological consequences of cross-immunity. Am J Public Health 1997;87:1923-7.

51. Rueda G. Tuberculosis. El triunfo de la medicina sobre la magia. En: Pérez M, Otero-Ruiz E, editores. El arte de curar. Un viaje a través de la enfermedad en Colombia 1898-1998. Bogotá: Afidro; 1998. p.91-102.

52. Romero A. Historia de la medicina en Colombia. Siglo XIX. Medellín: Universidad de Antioquia-Colciencias; 1996 p.209.

53. Hernández $M$. La salud fragmentada en Colombia, 1910-1946. Bogotá: Instituto de Salud Pública, Universidad Nacional de Colombia; 2002.

54. Rueda G. Apuntaciones sobre la historia de la tuberculosis. Rev Colomb Neumol 1991;3:15-9.

55. Maldonado $\mathbf{H}$, Hernández $\mathbf{M}$. Memorias de un sanatorio antituberculoso. Biomédica 2004;24(supl):27-33.

56. Elzinga G, Raviglione MC, Maher D. Scale up: meeting targets in global tuberculosis control. Lancet 2004; 363:814-9.

57. Dubos R, Dubos J. The white plague: Tuberculosis, man, and society. $2^{\text {nd }}$ ed. New Brunswick: Rutgers University Press; 1987.

58. Gonçalves H. A tuberculose ao longo dos tempos. Hist Cienc Saúde Manguinhos 2000;7:303-25.

59. Ferreira-Antunes JL, Waldman EA. Tuberculosis in the twentieth century: time series mortality in São Paulo, Brazil, 100-97. Cad Saúde Pública 1999;15:463-76.

60. Paluzzi JE. A social disease/a social response: lessons in tuberculosis from early 20th century Chile. Soc Sci Med 2004;59:763-73.

61. Cohen ML. Changing patterns of infectious disease. Nature 2000;406:762-7.

62. Ospina S. La tuberculosis, una perspectiva históricoepidemiológica. Infectio 2001;5:241-50.

63. Sivigila. Tuberculosis. Hechos en vigilancia en salud pública. Sivigila 2001; semana epidemiológica No.
46, noviembre 11 al 17 de 2001. Disponible en: http:// www.col.ops-oms.org/sivigila/2001/BOLE46_2001.htm.

64. Sarmiento MC. Enfermedades transmisibles en Colombia: cambios ambivalentes. Rev Salud Pública (Bogotá) 2000;2:82-93.

65. Guerrero MI, León CI, De la Hoz F. The epidemiological evidence for an association between extrapulmonary tuberculosis and AIDS in Colombia. Tubercle and Lung Diseases 1994;75:160-1.

66. Murcia MI, Gómez JE, Alvarado F, Bustillo FG, Mendivelson E, Gómez B et al. Frequency of tuberculous and non-tuberculous mycobacteria in HIV infected patients form Bogotá, Colombia. BMC Infectious Diseases 2001;1:21.

67. Orozco LC, Aparicio G, Quintero O, Giraldo E, Ulloa I, León Cl. Resistencia de M. tuberculosis a las drogas. Biomédica 1981;1:130-4.

68. Ayala-Cerna C, Kroeger A. La reforma del sector salud en Colombia y sus efectos en los programas de control de tuberculosis e inmunización. Cad Saúde Pública 2002;18:1771-81.

69. Jaramillo E. Tuberculosis and stigma: predictors of prejudice against people with tuberculosis. J Health Psychol 1999;4:71-9.

70. Jaramillo E. Pulmonary tuberculosis and healthseeking behaviour: how to get a delayed diagnosis in Cali, Colombia. Trop Med Int Health 1998;3:138-44.

71. Cáceres DC, Izquierdo VF, Mantilla L, Jara J, Velandia M. Perfil epidemiológico de la población desplazada por el conflicto armado interno del país en un barrio de Cartagena, Colombia, 2000. Biomédica 2002;22:42544.

72. Vynnycky E, Fine PEM. Interpreting the decline in tuberculosis: the role of secular trends in effective contact. Int J Epidemiol 1999;28:327-34.

73. Krieger N. Epidemiology and the web of causation: Has anyone seen the spider? Soc Sci Med 1994;39:88793.

74. Jaramillo E. Encompassing treatment with prevention: The path for a lasting control of tuberculosis. Soc Sci Med 1999;49:393-404. 